\title{
Symptomatic Sinus Pericranii with Adult Onset Headache : A Case Report with Pathologic Perspective
}

\author{
Young Soo Chung', Jung-jae Kim², Se Hoon Kim³, Joonho Chung', Jae Whan Lee', Keun Young Park' \\ 'Department of Neurosurgery and ${ }^{3}$ Pathology, Severance Hospital, Yonsei University College of Medicine, Seoul, Korea ; \\ ${ }^{2}$ Department of Neurosurgery, Ewha Womans University Seoul Hospital, Ewha Womans University School of Medicine, \\ Seoul, Korea
}

Sinus pericranii (SP) is a rare vascular anomaly of the scalp that consists of an abnormal pericranial venous channel connected to adjacent dural venous sinuses. Most SP are asymptomatic and are found in the pediatric age group. We aim to report a case of symptomatic SP in adult and describe the clinical, radiological, and pathohistological findings to help understand and differentiate this lesion from other scalp lesions. A 40-year-old man with a scalp mass was admitted to our hospital complaining of headache. The lesion enlarged when the patient was in a recumbent position or during Valsalva maneuver. The radiologic imaging suggested its diagnosis as an accessory type of SP with bone erosion. Surgical resection and cranioplasty were successfully performed, and the related headache also gradually subsided. At the 3-year follow-up, there was no recurrence on magnetic resonance imaging.

Keywords Sinus pericranii; Vascular malformation; Headache
J Cerebrovasc Endovasc Neurosurg. 2019 September;21(3):163-168

Received : 9 July 2019

Revised : 16 September 2019

Accepted : 20 September 2019

\begin{abstract}
Correspondence to Keun Young Park Department of Neurosurgery, Severance Hospital, Yonsei University College of Medicine, 50 Yonsei-ro, Seodaemun-gu, Seoul 120-752. Republic of Korea

Tel : 82-2-2228-2150

Fax : 82-2-393-9979

E-mail : kypark78.md@gmail.com

ORCID : http://orcid.org/0000-0002-3254-4577
\end{abstract}

This is an Open Access article distributed under the terms of the Creative Commons Attribution NonCommercial License (http://creativecommons.org/licenses/by-nc/3.0) which permits unrestricted noncommercial use, distribution, and reproduction in any medium, provided the original work is properly cited.

\section{INTRODUCTION}

Sinus pericranii (SP) is a rare vascular anomaly, which forms as a scalp varix consisting of an extracranial-intracranial venous communication with or without a skull defect. ${ }^{9)}$ Generally, SP is a benign lesion and presents as an asymptomatic mass in childhood. ${ }^{11)}$ Sometimes, its rarity and similarity with other scalp lesions can lead to misdiagnosis of SP as a scalp hemangioma, ${ }^{2)}$ atretic cephalocele, ${ }^{3)}$ or other scalp vascular anomalies. However, its unique characteristics are helpful to differentiate a SP from other vascular anomalies. Herein, we describe the clinical, radiological, and pathohistological findings of a case of SP to help understand this rare disease entity.

\section{DESCRIPTION OF CASE}

A 40-year-old man with a mass on the vertex visited our clinic. In his medical history, the mass had existed since his childhood without any change in size. However, the mass had grown gradually in the recent several months, and a newly developed headache occurred. The headache was characterized with a persistent waxing and waning, and relieved using analgesics. Due to this headache, he was distressed while working. Apart from headache, there was no other symptom related with the lesion. The mass was soft, round-shaped, and non-tender lesion. Characteristically, its size increased when in a recumbent position and during Valsalva maneuver, and returned to its original size in a standing position. 


\section{Radiologic findings}

The initial computed tomography (CT) imaging showed a slight high-density scalp mass with a related bone erosion of the calvarial outer table. The inner table was very thin; however, it was not completely destroyed. The lesion was located in the midline, adjacent to the superior sagittal sinus (Fig. 1a, b). On magnetic resonance (MR) imaging, a scalp mass with heterogenous signals and flow-void in both $\mathrm{T} 1$ and T2 weighted imaging was identified. Entangled morphology and enhancement after gadolinium contrast implied a vascular anomaly (Fig. 2a, b, c). No definite brain parenchymal lesion was seen. On the venous phase of digital subtraction angiography (DSA), small vascular channel was noted on right carotid angiogram, and this anomaly was connected to the superior sagittal sinus (SSS). While a majority of the cerebral venous outflow occurred through the SSS, only a part of the extracranial venous outflow was draining through the SP (Fig. 3), implying "accessory type" of SP. ${ }^{4)}$ There were no other vascular anomalies such as arteriovenous malformation or cavernous malformation. These clinical and radiologic findings strongly suggested a tentative SP.

\section{Operative findings}

As a definite treatment, surgical excision was performed. Under general anesthesia, the scalp was meticulously dissected with coagulation of small scalp vessels in a layer by layer fashion. The reddish, hyperemic, and soft lesion was located on the calvarium. Coagulation and cutting of the small vessels around the lesion were performed using a bipolar coagulator, then the lesion was carefully dissected from a nearby soft tissue and bone. Finally, the lesion was completely removed. During the surgery, a lot of small holes were identified within the calvarium. These holes formed tiny channels connected with the SSS; some hemorrhagic leakage occurred from them. After achieving hemostasis using a bone wax and a glue, cranioplasty was done using a mesh plate system. These surgical procedures were performed without any complications.

\section{Postoperative clinical course}

Immediate postoperative CT imaging showed a complete removal of SP and a well-formed cranial vault (Fig. 1c). At discharge, the patient had completely recovered. At the 3-year follow-up, there was no evidence of disease recurrence on MR imaging (Fig. 2d) and the patient was doing well without any headache and neurologic signs.

\section{Pathologic findings}

A thin endothelial lining with CD-31(vascular marker) positive and D2-40 (lymphatic marker) negative histology was identified; small, multiple void chan-
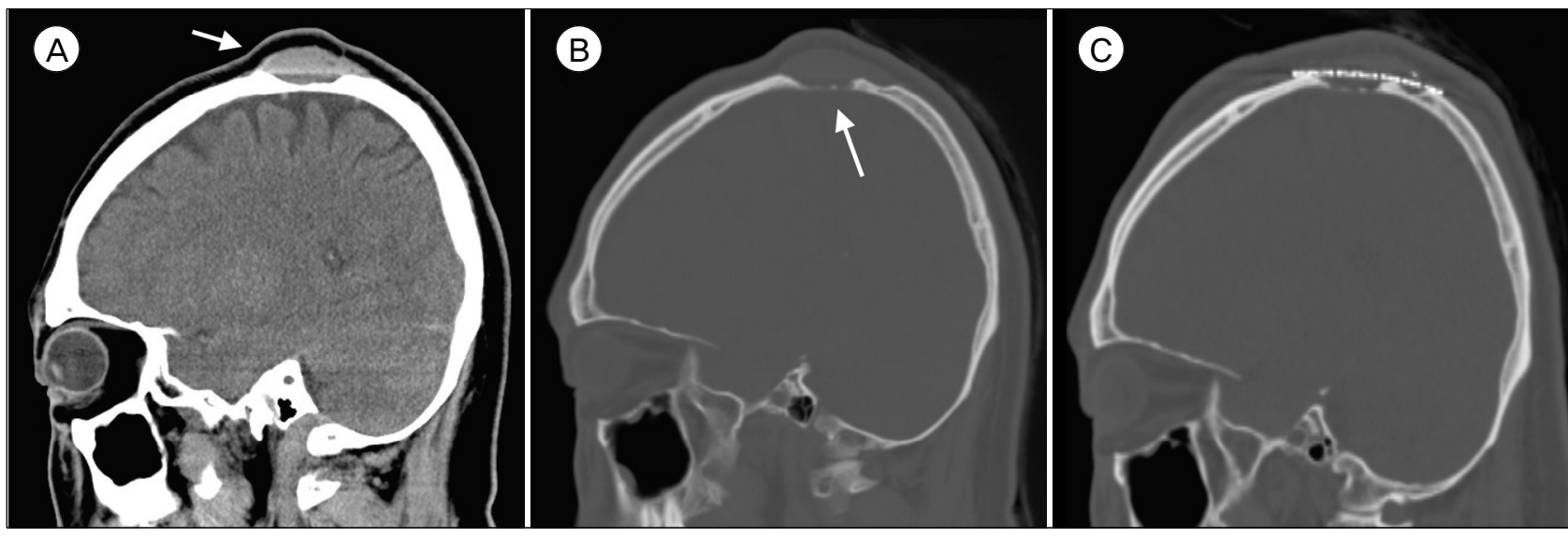

Fig. 1. Computed tomography (CT) scan. (A) Sagittal view, isodense round-shaped mass (arrow) located above the skull. (B) Sagittal view of bone window setting, the related calvarial erosion (arrow) was noted. (C) Complete removal of sinus pericranii and well-formed cranial vault with mesh plate was noted in postoperative CT scan. 

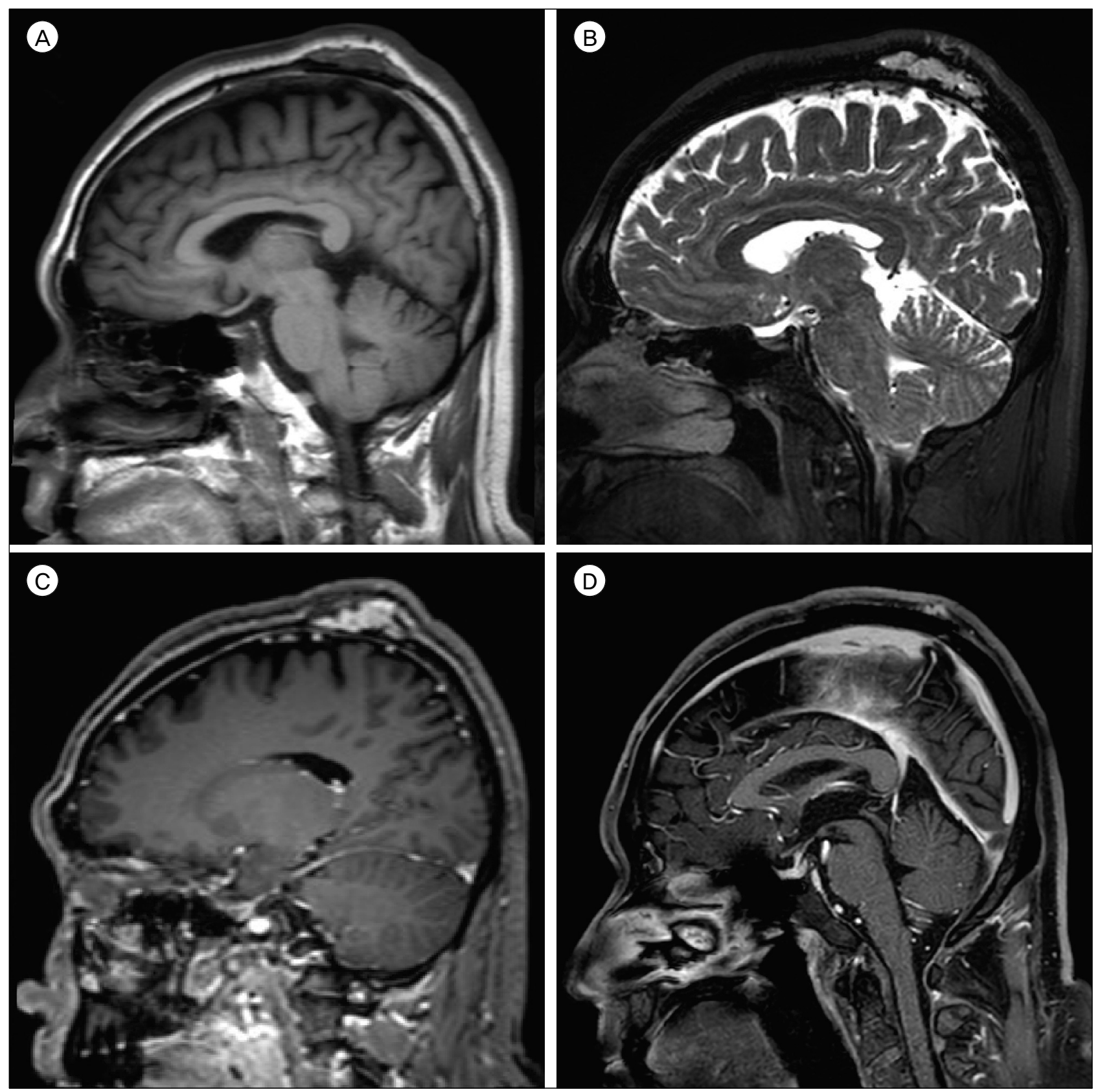

Fig. 2. Preoperative magnetic resonance (MR) imaging showed heterogenous signals and flow voids within the mass in both $\mathrm{T1}(\mathrm{A})$ and T2 (B) weighted images. Gadolinium-enhanced T1-weighted MR imaging (C) showed a contrast-enhancing mass between galea aponeurotica and skull. Three year's follow-up gadolinium-enhanced T1-weighted MR imaging (D) showed a complete obliteration of mass without any recurrence.

nels were well described. A thick fibrous stroma intervening endothelium indicated its presence for several years, which indicated a possibility of congenital type SP (Fig. 4). Unlike cavernous hemangioma, interwoven capillary with hemorrhage was not found. Furthermore, there was no neural or meningeal tissue, which is seen in encephalocele. No thrombus was noted.

\section{DISCUSSION}

Sinus pericranii (SP) is a kind of scalp vascular lesion characterized by an extracranial-intracranial ve- 


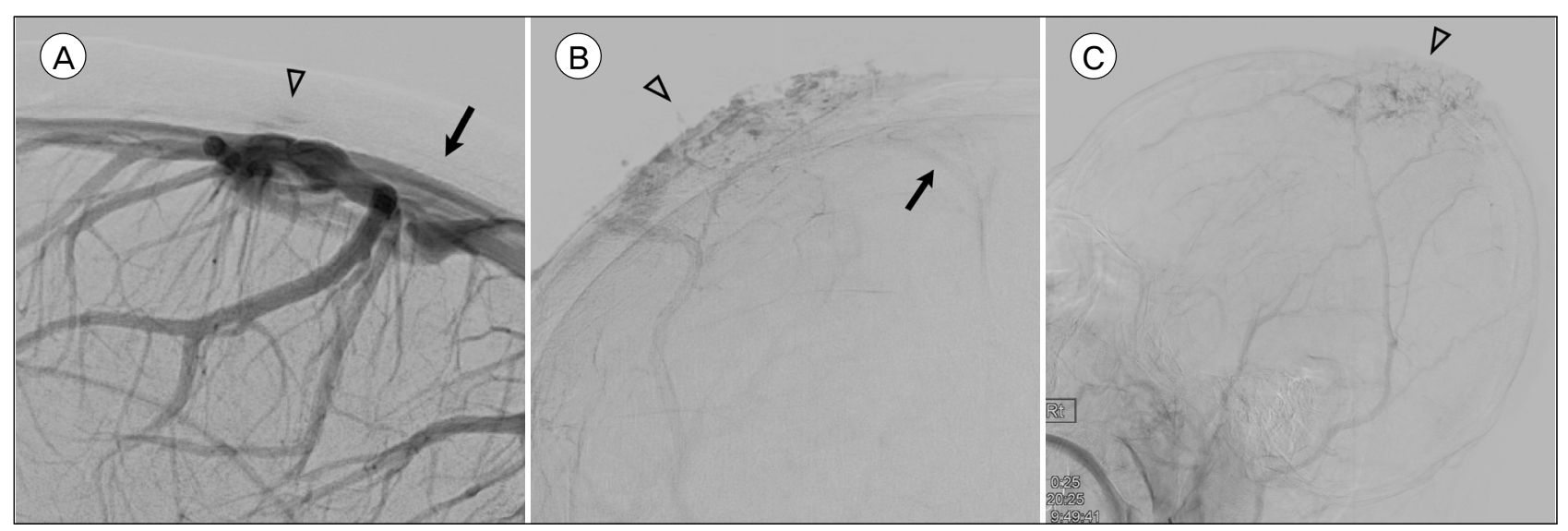

Fig. 3. Digital subtraction angiography (DSA) findings of sinus pericranii (SP). (A) Internal carotid angiography lateral view; in late venous phase, a majority of the cerebral venous outflow occurred through the superior sagittal sinus (SSS, arrow). Small contrast filling of extracranial-intracranial venous channel was also identified (arrowhead). (B) External carotid angiography AP view; in late venous phase, the SP (arrowhead) was connected with SSS (arrow). (C) External carotid angiography lateral view; in late venous phase, only a part of the extracranial venous outflow was draining through the SP (arrowhead). These findings indicated an accessory type SP.

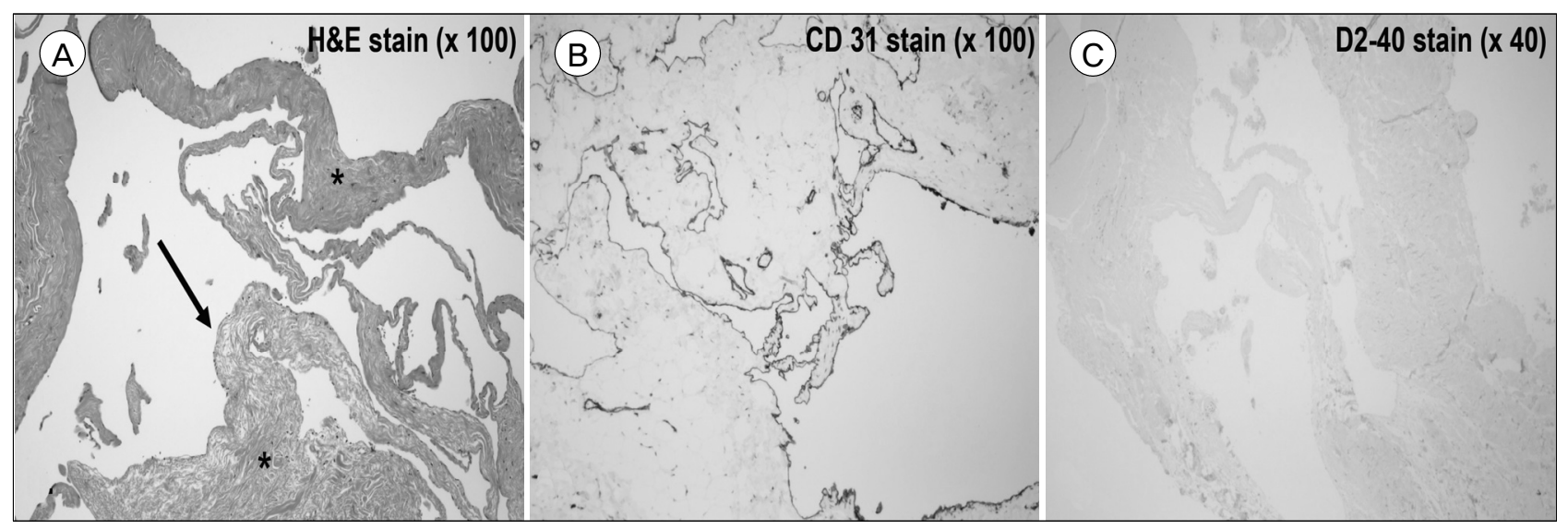

Fig. 4. Pathologic findings of sinus pericranii (SP). (A) H\&E staining (x 100); it showed a single-layer flattened endothelium (arrow) with thick venous stroma (asterisk), which suggested a congenital type of SP. (B) CD-31 staining ( $x$ 100); thin brownish endothelial layers showed the vascular nature. (C) D2-40 staining (x 40); it showed the absence of lymphatic wall.

nous communication, which can be formed either by focal venous hypertension and abnormal development of diploic veins (congenital type) or by trauma (acquired type). ${ }^{110)}$ SP is very rare and unfamiliar to clinicians, thus, it can be easily misdiagnosed as other scalp diseases. However, SP has its own unique clinical and radiological features, ${ }^{614)}$ which can be helpful for diagnosis. Unlike other scalp pathology including vascular anomaly, SP is usually located along the midline $^{11)}$ and its size can fluctuate depending on body positioning or Valsalva maneuver. ${ }^{8)}$ Usually, SP can be associated with bone erosion, thus, CT or MR imaging shows a hyperemic vascular channeling in the scalp layer with the related bone destruction. DSA can be helpful for definitive diagnosis and deciding treatment option, through analyzing venous flow dynamics and discovering the vascular nature. ${ }^{7)}$ Some authors recommend direct percutaneous venography as a confirmatory tool, ${ }^{1)}$ however, it is associated with a high risk of bleeding and infarction.

Approximately $80 \%$ of SP present as an asymptomatic palpable mass. ${ }^{1)}$ Nevertheless, headache is a common feature of symptomatic SP. ${ }^{8113)}$ The SP-related headache usually has a waxing and waning pattern 
for several months, however, it can also present as a sudden striking headache in some cases. Although the pathophysiology of SP-related headache is uncertain, intracranial hypertension can be one of the causes of the headache." Therefore, clinicians should keep in mind that SP is one of the possible causes of headache and intracranial hypertension.

Because most SP has benign features, asymptomatic SP can be observed or conservatively treated. On the other hand, active treatment should be considered in a symptomatic or cosmetically problematic SP. However, all symptomatic SP cannot be actively treated. Using the venous drainage pattern, SP can be classified into a dominant type or an accessory type. ${ }^{4)}$ The dominant type of SP is defined when a majority of the venous outflow occurs through the SP. Meanwhile, when only a small portion of the venous drainage flows through the SP, it is called as the accessory type. In the dominant type, active treatment should be avoided because of the consequential venous infarction and bleeding. On the contrary, the accessory type of SP can be treated by surgical resection or endovascular embolization. ${ }^{12)}$ In our case, the SP was symptomatic one and its venous outflow was marked via the SSS not through the SP, suggesting an accessory type. Thus, it was treated by a single session of surgery and the headache was also relieved.

Most of SP is a congenital type, which formed at late embryogenesis. After birth, the lesion which has been for several years can have a typical feature of pathologic findings. The existence of thick accumulated stroma suggested a possibility of congenital type SP. On the other hand, acquired type SP usually depicts fibrous microstructure encapsulating the blood. This is because trauma disrupts emissary veins and calvarium, making a fibrous lining or capsule around the extravasated blood. The lesion described here is regarded as a congenital type due to its characteristic history and pathologic findings.

\section{CONCLUSION}

Sinus pericranii (SP) is known as a rare vascular anomaly and it can be easily misdiagnosed. However, its unique clinical and radiological characteristics can be helpful as regards differential diagnoses and treatment decision.

\section{Disclosure}

The authors have no conflict of interest concerning this case report.

\section{REFERENCES}

1. Akram H, Prezerakos G, Haliasos N, O'Donovan D, Low H. Sinus pericranii: an overview and literature review of a rare cranial venous anomaly (a review of the existing literature with case examples). Neurosurg Rev. 2012 Jan;35(1):15-26; discussion

2. Arita K, Uozumi T, Kuwabara S, Kiya K, Sumida M, Iida $\mathrm{K}$ et al. A case of scalp cavernous hemangioma simulating sinus pericranii. Hiroshima J Med Sci. 1992 Mar;41(1):19-23.

3. Bick DS, Brockland JJ, Scott AR. A scalp lesion with intracranial extension. Atretic cephalocele. JAMA Otolaryngol Head Neck Surg. 2015 Mar;141(3):289-90.

4. Gandolfo C, Krings T, Alvarez H, Ozanne A, Schaaf M, Baccin CE et al. Sinus pericranii: diagnostic and therapeutic considerations in 15 patients. Neuroradiology. 2007 Jun;49(6):505-14.

5. Guler S, Tatli B. Rare vascular pathology sinus pericranii; becomes symptomatic with pseudotumor cerebri Turk J Pediatr. 2015 Nov-Dec;57(6):618-20.

6. Kaido T, Kim YK, Ueda K. Diagnostic and therapeutic considerations for sinus pericranii. J Clin Neurosci. 2006 Aug;13(7):788-92.

7. Khachatrian VA, Khodorovskaia AM, Sebelev KI, Zabrodskaia IuM. Pericranial sinus. Definition, diagnosis, surgical treatment. Zh Vopr Neirokhir Im N N Burdenko. 2014;78(3):30-7.

8. Lee $\mathrm{CH}$, Lee YS, Lee JH, Lee HG, Ryu KY, Kang DG. Sinus pericranii: A case report and the literature review. Korean J CerebrovascSurg. 2009 Dec;11(4):174-8.

9. Nomura S, Kato S, Ishihara $H$, Yoneda $H$, Ideguchi $M$, Suzuki M. Association of intra- and extradural developmental venous anomalies, so-called venous angioma and sinus pericranii. Childs Nerv Syst. 2006 Apr;22(4): $428-31$.

10. Ota T, Waga S, Handa H, Nishimura S, Mitani T. Sinus pericranii. J Neurosurg. 1975 Jun;42(6):704-12.

11. Pavanello M, Melloni I, Antichi E, Severino M, Ravegnani M, Piatelli $G$ et al. Sinus pericranii: diagnosis and management in 21 pediatric patients. J Neurosurg Pediatr. 2015 Jan;15(1):60-70. 
12. Rangel-Castilla L, Krishna C, Klucznik R, Diaz O. Endovascular embolization with Onyx in the management of sinus pericranii: a case report. Neurosurg Focus. 2009 Nov;27(5):E13.

13. Saba R, Senussi MH, Alwakkaf A, Brown H. Sinus peri- cranii in a young adult with chronic headache. BMJ Case Rep. 2013 Jul;2:2013.

14. Sadler LR, Tarr RW, Jungreis CA, Sekhar L. Sinus pericranii: CT and MR findings. J Comput Assist Tomogr. 1990 Jan-Feb;14(1):124-7. 